05

\title{
Влияние редкоземельных металлов на стеклообразующую способность и кристаллизацию аморфных сплавов CoFeSiBNb
}

\author{
(C) Б.А. Русанов, ${ }^{1}$ B.E. Сидоров, ${ }^{1,2}$ P. Svec, ${ }^{3}$ D. Janickovic, ${ }^{3}$ B.И. Ладьянов, ${ }^{4}$ C.A. Петрова, ${ }^{2,5}$ A.А. Сабирзянов ${ }^{6}$ \\ ${ }^{1}$ Уральский государственный педагогический университет, \\ 620017 Екатеринбург, Россия \\ 2 Уральский фредеральный университет, \\ 620002 Екатеринбург, Россия \\ ${ }^{3}$ Institute of Physics, Slovak Academy of Sciences, \\ 84511 Bratislava, Slovakia \\ ${ }^{4}$ Удмуртский фредеральный исследовательский центр УрО РАН, \\ 426067 Ижевск, Россия \\ ${ }^{5}$ Институт металлургии УрО РАН, \\ 620016 Екатеринбург, Россия \\ ${ }^{6}$ Уральский государственный университет путей сообщения, \\ 620034 Екатеринбург, Россия \\ e-mail: rusfive@mail.ru
}

Поступило в Редакцию 30 мая 2021 г.

В окончательной редакции 21 сентября 2021 г.

Принято к публикации 22 сентября 2021 г.

Получены аморфные сплавы $\mathrm{Co}_{48} \mathrm{Fe}_{25} \mathrm{Si}_{4} \mathrm{~B}_{19} \mathrm{Nb}_{4}-\mathrm{P} 3 \mathrm{M}(\mathrm{P} 3 \mathrm{M}=\mathrm{Nd}, \mathrm{Sm}, \mathrm{Tb}, \mathrm{Yb})$ в виде лент шириной 3-5 mm и толщиной 37-40 $\mu \mathrm{m}$. Методом дифференциального термического анализа установлено, что кристаллизация этих сплавов идет, как правило, в два этапа и зависит от используемого редкоземельного металла и его содержания в сплаве. Рассчитаны критерии стеклообразующей способности сплавов. Показано, что парамагнитная температура Кюри для сплавов в жидком состоянии может быть использована как априорный критерий их стеклообразующей способности.

Ключевые слова: аморфные сплавы, кристаллизация, стеклообразующая способность, дифференциальный термический анализ, дифракция рентгеновских лучей, редкоземельные металлы.

DOI: 10.21883/JTF.2022.01.51852.165-21

\section{Введение}

Объемно-аморфные металлические сплавы являются объектами с уникальными механическими, магнитными и коррозионными свойствами [1]. Эти материалы уже нашли промышленное применение. В частности, аморфные проволоки на основе кобальта и железа используются в качестве новых высокочувствительных сенсорных датчиков [2-4]. Однако широкое применение кобальтовых объемно-аморфных материалов ограничено их достаточно низкой стеклообразующей способностью. Поэтому вопрос повышения их стеклообразующей способности остается актуальным.

Не так давно были получены новые объемно-аморфные сплавы составов $\mathrm{Co}_{46} \mathrm{Fe}_{20} \mathrm{~B}_{22-x} \mathrm{Si}_{6-x} \mathrm{Nb}_{6}$

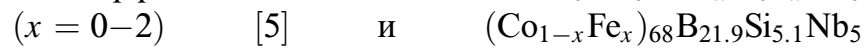
$(x=0.1-0.5)$ [6] в виде цилиндров диаметром $5.5 \mathrm{~mm}$. Согласно авторам, эти металлические стекла обладают прекрасными магнитными свойствами (намагниченность насыщения достигает значений 0.49-0.91 T при коэрцитивной силе $0.71-1.58 \mathrm{~A} / \mathrm{m}$, а эффективная проницаемость равна $(2.21-3.25) \cdot 10^{4}$ при $1 \mathrm{kHz}$ в поле напряженностью $1 \mathrm{~A} / \mathrm{m})$. Кроме того, эти объемноаморфные сплавы на основе кобальта характеризуются высокими значениями предела прочности на сжатие на уровне $4250-4450 \mathrm{MPa}$ и пластической деформации в $0.6-1.3 \%$ [5-7]. Наши предыдущие исследования показали $[8,9]$, что стеклообразующую способность сплавов на основе кобальта можно увеличить за счет введения небольших добавок легирующих элементов, удовлетворяющих критериям, сформулированным А. Inoue [1]. Применение других легирующих добавок для улучшения стеклообразующей способности сплавов на основе железа и кобальта обсуждается в работах [10,11].

В настоящей работе мы использовали добавки 1 и 2 at.\% редкоземельных (РЗМ) металлов $\mathrm{Nd}, \mathrm{Sm}, \mathrm{Tb}$, $\mathrm{Yb}$ к базовой композиции $\mathrm{Co}_{48} \mathrm{Fe}_{25} \mathrm{Si}_{4} \mathrm{~B}_{19} \mathrm{Nb}_{4}$ и изучили кристаллизацию аморфных лент методом дифференциального термического анализа (ДТА), а также провели расчет критериев стеклообразующей способности указанных сплавов.

\section{1. Методика эксперимента}

Сплавы составов $\mathrm{Co}_{48} \mathrm{Fe}_{25} \mathrm{Si}_{4} \mathrm{~B}_{19} \mathrm{Nb}_{4}$ (базовый состав (master alloy) - MA) и с малыми добавками редкоземельных металлов ( $\mathrm{Nd}, \mathrm{Sm}, \mathrm{Tb}, \mathrm{Yb})$ приготовлены переплавкой исходных компонентов в индукционной 
печи при температуре $1900 \mathrm{~K}$ в течение получаса в атмосфере аргона.

Аморфные ленты шириной 3-5 mm и толщиной 37-40 $\mu \mathrm{m}$ получены методом спиннингования в контролируемой атмосфере аргона после предварительного перегрева расплавов до $1700-1723 \mathrm{~K}$ в индукционной печи и последующего инжектирования на вращающийся водоохлаждаемый медный барабан. Образцы были приготовлены в виде лент для удобства измерения их электросопротивления и ДТА.

Структура образцов проанализирована методом дифракции рентгеновских лучей на дифрактометре Bruker D8 Advance (CuK $\alpha$-излучение). Кинетика кристаллизации аморфных сплавов изучена с помощью дифференциального термического анализа на установке PerkinElmer DTA-7 со скоростью нагрева $10 \mathrm{~K} / \mathrm{min}$. В качестве образца сравнения использован оксид алюминия $\mathrm{Al}_{2} \mathrm{O}_{3}$. Предварительно установку калибровали по точкам плавления чистых алюминия и золота, погрешность определения температуры составила $\pm 1 K$.

\section{2. Результаты и обсуждение}

Дифрактограммы МА и полученных в виде лент сплавов с добавками редкоземельных металлов представлены на рис. 1.

Из рис. 1 видно, что все полученные образцы являются рентгеноаморфными. Обнаружено, что в образцах с неодимом и иттербием (1 at.\%) на дифрактограммах присутствует предпик, расположенный левее основного пика. Аналогичный результат получен для аморфных сплавов, содержащих 2 at.\% тербия и иттербия. При этом величина обнаруженного предпика максимальна в сплаве с тербием. Сплавы с самарием таких особенностей не имеют. Наличие предпика может свидетельствовать как о существовании среднего порядка в сплавах, так и о наличии микрообластей, обогащенных одним из компонентов, т. е. о микрогетерогенности сплавов.

С помощью ДТА исследована кинетика кристаллизации аморфных образцов. Кривые базовой композиции (МА), снятые при различных скоростях нагрева, приведены на рис. 2.

Обнаружено, что базовая композиция имеет одну экзотермическую реакцию при переходе из аморфного состояния в кристаллическое. Процесс же плавления базовой композиции занимает широкий температурный интервал и сопровождается двумя эндотермическими реакциями.

Кривые ДТА, полученные для сплавов с добавками редкоземельных металлов, приведены на рис. 3 .

Установлено, что термограммы ДТА характеризуются одним или двумя экзотермическими пиками в зависимости от используемого РЗМ. В исследованных составах не обнаружено температур стеклообразного перехода (glass-transition temperature $-T_{g}$ ). Наряду с этим, добавки РЗМ уменьшают температурный интервал плавления
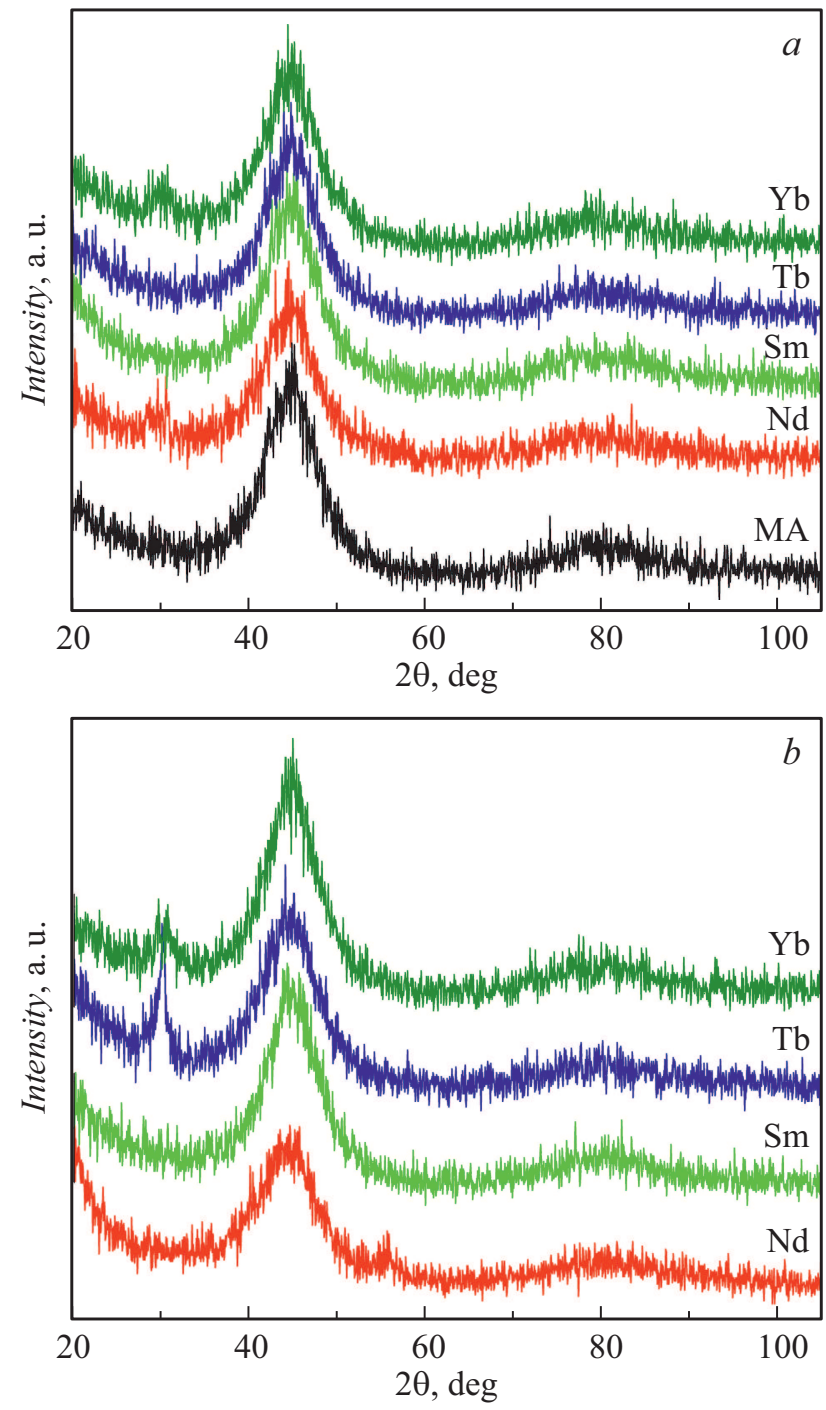

Рис. 1. Дифрактограммы аморфных лент $\mathrm{Co}_{48} \mathrm{Fe}_{25} \mathrm{Si}_{4} \mathrm{~B}_{19} \mathrm{Nb}_{4}-\mathrm{P} 3 \mathrm{M} \quad(\mathrm{P} 3 \mathrm{M}=\mathrm{Nd}, \quad \mathrm{Sm}, \quad \mathrm{Tb}, \quad \mathrm{Yb})$. $a$ - базовый состав (MA) и сплавы, содержащие 1 at.\% Р3М, $b$ - сплавы, содержащие 2 at.\% Р3М. Кривые смещены по вертикали для наглядности.

$T_{L}-T_{S}$ в исследованных сплавах (для базового состава он составляет $134 \mathrm{~K}$ ). Характеристические температуры для исследованных сплавов представлены в табл. 1 .

Фазовый состав полностью закристаллизованных образцов оказался следующим (для базового состава): ГЦК-кобальт и ОЦК-раствор $\mathrm{Co}-\mathrm{Fe}$ (микрообласти с разной концентрацией) - 52\%; оксиды (преимущественно $\mathrm{Fe}_{2.2} \mathrm{Co}_{0.8} \mathrm{O}_{4}$ и $\left.\mathrm{CoO}\right)$ - 13\%; сложные $\left(\mathrm{Fe}_{3} \mathrm{Co}_{3} \mathrm{~B}_{2}, \mathrm{Fe}_{3} \mathrm{Si}_{0.4} \mathrm{~B}_{0.6}\right)$ и простые $\left(\mathrm{Co}_{2} \mathrm{~B}, \mathrm{Fe}_{2} \mathrm{~B}\right)$ бориды - 29\%; метастабильная фаза 23:6 (преимущественно $\left.\mathrm{Co}_{11.2} \mathrm{Fe}_{9.8} \mathrm{Nb}_{2} \mathrm{~B}_{6}\right)-6 \%$.

$\mathrm{C}$ добавлением редкоземельных металлов фазы $\mathrm{Co}-\mathrm{Fe}$ становится существенно больше (до 65\%), а оксидов и боридов - существенно меньше (4 и 20\% соответственно). Кроме того, в сплавах с неодимом, иттербием 


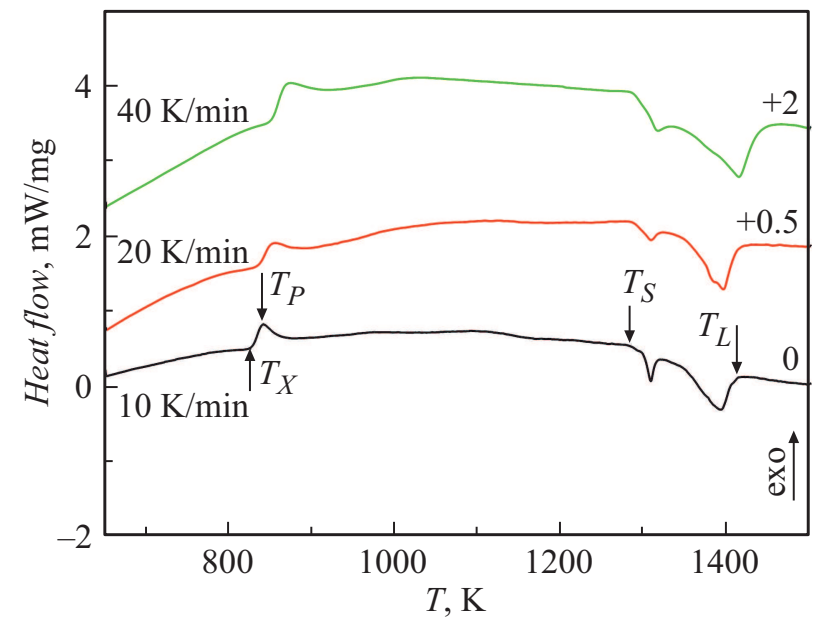

Рис. 2. Кривые ДТА аморфного сплава $\mathrm{Co}_{48} \mathrm{Fe}_{25} \mathrm{Si}_{4} \mathrm{~B}_{19} \mathrm{Nb}_{4}$, снятые при различных скоростях нагрева. Стрелками показаны характеристические температуры: $T_{X}$ - температура начала кристаллизации, $T_{P}-$ температура пика, $T_{S}-$ солидус, $T_{L}-$ ликвидус. Цифры указывают величину смещения кривых по вертикали.

Таблица 1. Характеристические температуры сплавов $\mathrm{CoFeSiBNb}-\mathrm{P} 3 \mathrm{M}$

\begin{tabular}{l|c|c|c|c}
\hline \multicolumn{1}{c|}{ Сплав } & $T_{X 1}, \mathrm{~K}$ & $T_{X 2}, \mathrm{~K}$ & $T_{S}, \mathrm{~K}$ & $T_{L}, \mathrm{~K}$ \\
\hline $\mathrm{Co}_{48} \mathrm{Fe}_{25} \mathrm{Si}_{4} \mathrm{~B}_{19} \mathrm{Nb}_{4}-\mathrm{MA}$ & $825 \pm 1$ & - & $1280 \pm 1$ & $1414 \pm 1$ \\
$\mathrm{MA}+1$ at.\% Nd & $841 \pm 1$ & $1025 \pm 1$ & $1301 \pm 1$ & $1398 \pm 1$ \\
$\mathrm{MA}+2$ at.\% Nd & $858 \pm 1$ & $994 \pm 1$ & $1316 \pm 1$ & $1410 \pm 1$ \\
$\mathrm{MA}+1$ at.\% Sm & $833 \pm 1$ & - & $1299 \pm 1$ & $1394 \pm 1$ \\
$\mathrm{MA}+2$ at.\% Sm & $839 \pm 1$ & - & $1298 \pm 1$ & $1394 \pm 1$ \\
$\mathrm{MA}+1$ at.\% Tb & $830 \pm 1$ & - & $1298 \pm 1$ & $1390 \pm 1$ \\
$\mathrm{MA}+2$ at.\% Tb & $846 \pm 1$ & $1038 \pm 1$ & $1301 \pm 1$ & $1393 \pm 1$ \\
$\mathrm{MA}+1$ at.\% Yb & $831 \pm 1$ & $1047 \pm 1$ & $1296 \pm 1$ & $1402 \pm 1$ \\
$\mathrm{MA}+2$ at.\% Yb & $837 \pm 1$ & $1042 \pm 1$ & $1294 \pm 1$ & $1391 \pm 1$
\end{tabular}

и в сплаве с 2\% тербия зафиксированы бориды РЗМ, которые появляются, по-видимому, при температурах второго пика на термограммах ДТА.

Определенные по результатам опытов характеристические температуры позволяют рассчитать некоторые критерии стеклообразующей способности исследованных сплавов. Существует большое количество таких критериев, однако мы выбрали те, в которые не входит температура стеклования $T_{g}$, так как она не была зафиксирована в наших опытах, а именно: $\Delta T_{L}=T_{L}-T_{X} \quad[12], \quad \alpha=T_{X} / T_{L} \quad[13,14], \quad \omega_{J}=\left(T_{L}\left(T_{L}+\right.\right.$ $\left.\left.+T_{X}\right)\right) /\left(T_{X}\left(T_{L}-T_{X}\right)\right)[15]$. В табл. 2 приведены расчеты указанных критериев стеклообразующей способности.

Следует отметить, что существующие критерии не обладают предсказательной функцией. Для их определения требуется сначала получить сплав в аморфном состоянии, затем определить характеристические температуры с высокой точностью и лишь после этого становится возможным рассчитать критерии стеклообразующей способности. Тем не менее из полученных результатов можно сделать вывод о том, что критерий $\Delta T_{L}$ является наиболее информативным из трех рассчитанных (он сильно изменяется при введении легирующей добавки), и наиболее эффективна та добавка, которая приводит к существенному росту температуры $T_{X}$. Критерии $\alpha$ и $\omega_{J}$ слабо отражают различия между сплавами.
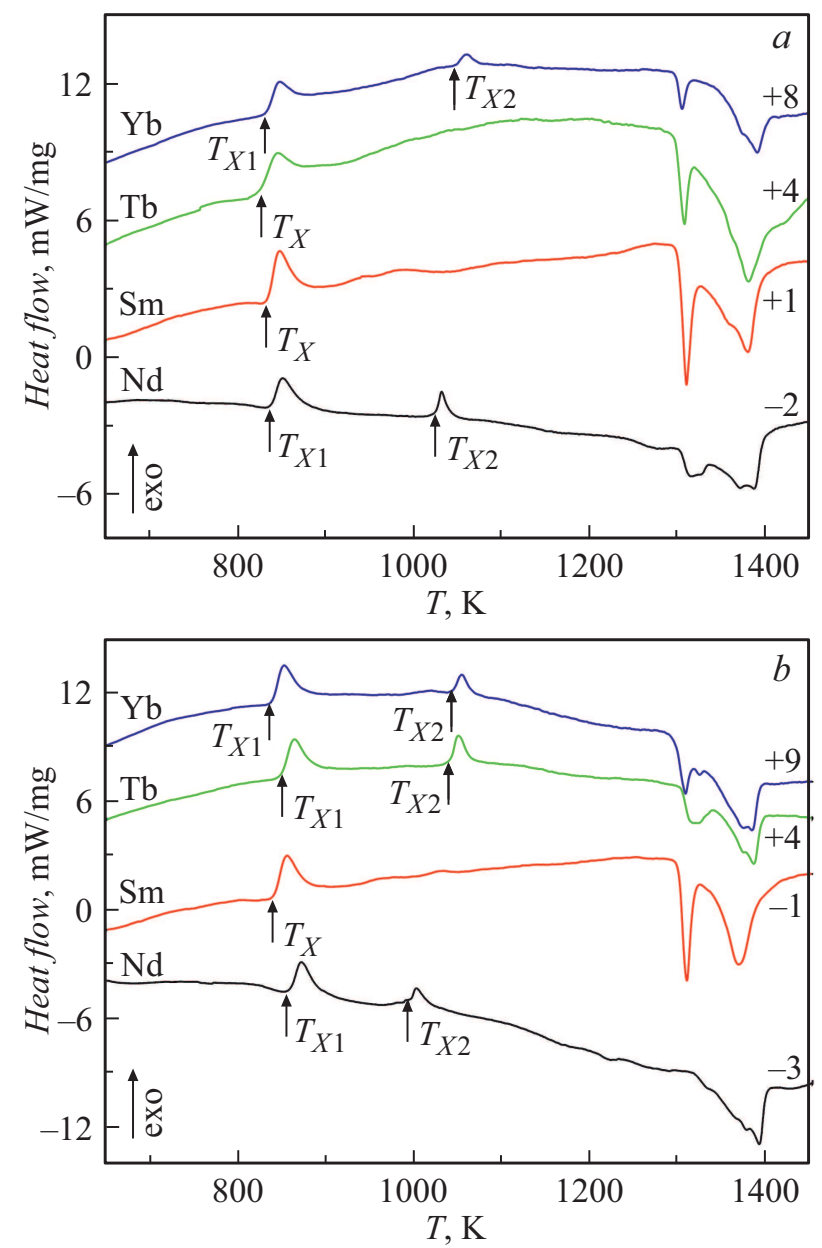

Рис. 3. Кривые ДТА сплавов $\mathrm{Co}_{48} \mathrm{Fe}_{25} \mathrm{Si}_{4} \mathrm{~B}_{19} \mathrm{Nb}_{4}-\mathrm{P} 3 \mathrm{M}$, полученные при скорости нагрева $10 \mathrm{~K} / \mathrm{min} . a-$ сплавы, содержащие 1 at.\% РЗМ, $b$ - сплавы, содержащие 2 at.\% РЗМ. Цифры указывают величину смещения кривых по вертикали.

Таблица 2. Критерии стеклообразующей способности сплавов $\mathrm{Co}_{48} \mathrm{Fe}_{25} \mathrm{Si}_{4} \mathrm{~B}_{19} \mathrm{Nb}_{4}-\mathrm{P} 3 \mathrm{M}$

\begin{tabular}{l|c|c|c|c}
\hline \multicolumn{1}{c|}{ Сплав } & $\Delta T_{L}, \mathrm{~K}[12]$ & $\alpha[13,14]$ & $\omega_{J}[15]$ & $\theta, \mathrm{K}[16]$ \\
\hline $\mathrm{Co}_{48} \mathrm{Fe}_{25} \mathrm{Si}_{4} \mathrm{~B}_{19} \mathrm{Nb}_{4}-\mathrm{MA}$ & 589 & 0.58 & 6.52 & 740 \\
$\mathrm{MA}+1$ at.\% Nd & 557 & 0.60 & 6.68 & 780 \\
$\mathrm{MA}+2$ at.\% Nd & 551 & 0.61 & 6.76 & 800 \\
$\mathrm{MA}+1$ at.\% Sm & 561 & 0.60 & 6.64 & 800 \\
$\mathrm{MA}+2$ at.\% Sm & 556 & 0.60 & 6.68 & 840 \\
$\mathrm{MA}+1$ at.\% Tb & 560 & 0.60 & 6.64 & 760 \\
$\mathrm{MA}+2$ at.\% Tb & 547 & 0.61 & 6.74 & 770 \\
$\mathrm{MA}+1$ at.\% Yb & 571 & 0.59 & 6.60 & 760 \\
$\mathrm{MA}+2$ at.\% Yb & 555 & 0.60 & 6.68 & 780
\end{tabular}


Ранее нами была выдвинута гипотеза об априорном определении стеклообразующей способности сплавов, склонных к аморфизации, на основе исследования их магнитных свойств в жидком состоянии и расчета парамагнитной температуры Кюри $(\theta)$ [8,16]. Температура $\theta$ фактически является обменным интегралом, т.е. характеризует силу межатомного взаимодействия в расплаве. Если легирующая добавка увеличивает $\theta$, то это означает, что она стабилизирует структуру расплава и препятствует его кристаллизации, т.е. улучшает стеклообразующую способность. В работе [16] высказано предположение о том, что наилучшими добавками для повышения стеклообразующей способности сплавов $\mathrm{Co}_{48} \mathrm{Fe}_{25} \mathrm{Si}_{4} \mathrm{~B}_{19} \mathrm{Nb}_{4}-\mathrm{P} 3 \mathrm{M}$ являются неодим и самарий. Результаты, приведенные в настоящей работе, подтверждают эту гипотезу: аморфные сплавы с неодимом и самарием характеризуются более равномерным распределением элементов по объему и повышенными значениями температур начала кристаллизации.

\section{Заключение}

Проведенные исследования ДТА аморфных сплавов $\mathrm{Co}_{48} \mathrm{Fe}_{25} \mathrm{Si}_{4} \mathrm{~B}_{19} \mathrm{Nb}_{4}-\mathrm{P} 3 \mathrm{M}$ показали, что все РЗМ повышают температуру начала кристаллизации. Рассчитанные критерии стеклообразующей способности вместе с данными о парамагнитной температуре Кюри позволяют заключить, что гипотеза об использовании этой температуры в качестве априорного критерия стеклообразующей способности подтвердилась.

\section{Благодарности}

P. Svec Sr. и D. Janickovic признательны за поддержку проектов VEGA 2/0144/21, APVV-19-0369.

\section{Конфликт интересов}

Авторы заявляют, что у них нет конфликта интересов.

\section{Список литературы}

[1] C. Suryanarayana, A. Inoue. Bulk Metallic Glasses (CRC Press, Boca Raton, 2017), DOI: 10.1201/9781315153483

[2] K. Mohri, K. Kawashima, T. Kozhawa, Y. Yoshida, L.V. Panina. IEEE Trans. Magn., 28, 3150 (1992). DOI: $10.1109 / 20.179741$

[3] L.V. Panina, K. Mohri, T. Uchiyama, M. Noda, K. Bushida. IEEE Trans. Magn., 31, 1249 (1995). https://doi.org/10.1109/20.364815

[4] H.Q. Guo, H. Kronmuller, T. Dragon, Z.H. Cheng, B.G. Shen. J. Appl. Phys., 89, 514 (2001). DOI: 10.1063/1.1331649
[5] Q. Man, H. Sun, Y. Dong, B. Shen, H. Kimura, A. Makino, A. Inoue. Intermetall., 18, 1876 (2010). DOI: $10.1016 /$ j.intermet.2010.02.047

[6] Y. Dong, A. Wang, Q. Man, B. Shen. Intermetall., 23, 63 (2012). DOI: $10.1016 /$ j.intermet.2011.12.020

[7] Q. Wang, G. Zhang, J. Zhou, C. Yuan, B. Shen. J. Alloys Compounds, 820, 153105 (2020). DOI: $10.1016 /$ j.jallcom.2019.153105

[8] V. Sidorov, J. Hosko, V. Mikhailov, I. Rozkov, N. Uporova, P. Svec,, D. Janickovic, I. Matko, P. Svec Sr, L. Malyshev. J. Magn. Magn. Mater., 354, 358 (2014). DOI: $10.1016 /$ j.jmmm.2013.10.038

[9] J. Hosko, I. Janotova, P. Svec, D. Janickovic, G. Vlasak, E. Illekova, I. Matko, P. Svec. J. Non-Crystall. Solids, $358(12-13), 1545$ (2012).

DOI: $10.1016 /$ j.jnoncrysol.2012.04.016

[10] Z.X. Dou, Y.L. Li, K. Lv, T. Wang, F.S. Li, X.D. Hui. Mater. Sci. Eng.: B, 264, 114942 (2021). DOI: $10.1016 /$ j.mseb.2020.114942

[11] M. Aykol, M.V. Akdeniz, A.O. Mekhrabov. Intermetall., 19 (9), 1330 (2011). DOI: 10.1016/j.intermet.2011.05.004

[12] Y. Zhang, D.Q. Zhao, M.X. Pan, W.H. Wang. Mater. Sci. Technol., 19 (7), 973 (2003). DOI: $10.1179 / 026708303225003045$

[13] T. Wakasugi, R. Ota, J. Fukunaga. J. Am. Ceram. Soc., 75 (11), 3129 (1992). DOI: 10.1111/j.1151-2916.1992.tb04398.x

[14] K. Mondal, B.S. Murty. J. Non-Cryst. Solids, 351 (16-17), 1366 (2005). DOI: 10.1016/j.jnoncrysol.2005.03.006

[15] X.L. Ji, Y. Pan. Trans. Nonferrous Met. Soc. China, 19(5), 1271 (2009). DOI: 10.1016/S1003-6326(08)60438-0

[16] Б.А. Русанов, В.Е. Сидоров, В.А. Михайлов, Р. Svec Sr., D. Janickovic. ЖТФ, 91 (8), 1253 (2021). DOI: $10.21883 /$ JTF.2021.08.51100.28-21 